\title{
Avaliação de potenciais interações medicamentosas em prescrições de antimicrobianos em um hospital no Estado do Pará
}

\author{
Evaluation of potential drug interactions in prescriptions of antimicrobials in a hospital in the State \\ of Pará
}

Evaluación de posibles interacciones medicamentosas en recetas de antimicrobianos en un hospital del Estado de Pará

Recebido: 14/04/2021 | Revisado: 21/04/2021 | Aceito: 21/04/2021 | Publicado: 08/05/2021

Samara de Oliveira Vilaça
ORCID: https://orcid.org/0000-0002-2710-3021
Universidade Federal do Pará, Brasil
E-mail: samaralivi@hotmail.com
Diego da Silva Santana
ORCID: https://orcid.org/0000-0002-2065-0073
Escola Superior da Amazônia-ESAMAZ, Brasil
E-mail: diegodssantana@ hotmail.com
Elizama da Sá Alves
ORCID: https://orcid.org/0000-0002-3444-1790
Escola Superior da Amazônia-ESAMAZ, Brasil
E-mail: elizama0sa@gmail.com
Soraia dos Santos Penha
ORCID: https://orcid.org/0000-0003-3907-1765
Escola Superior da Amazônia-ESAMAZ, Brasil
E-mail: sol_penha@yahoo.com.br
Valdicley Vieira Vale
ORCID: https://orcid.org/0000-0001-6570-4875
E-mail: valdicleyvale@ gmail.com
Universidade Federal do Pará/Escola superior da Amazonia-ESAMAZ, Brasil
Carolina Heitmann Mares Azevedo Ribeiro
ORCID: https://orcid.org/0000-0002-9457-2733
Universidade Federal do Pará, Brasil
E-mail: carolmheitman@hotmail.com

\begin{abstract}
Resumo
Objetivo: avaliar as potenciais interações medicamentosas de antimicrobianos em prescrições de pacientes internados em um hospital no Estado do Pará, no ano de 2019. Metodologia: Trata-se de estudo quali-quantitativo, retrospectivo e transversal, realizado através da coleta de dados de prontuários de pacientes que fizeram uso de antibióticos e estavam internados no ano de 2019 em um hospital no Pará. As interações medicamentosas foram avaliadas através da base de dados do Micromedex. Resultados: No período em estudo, 83\% ( $\mathrm{n}=762)$ dos pacientes que foram internados na instituição fizeram uso de antibiótico. Houve prevalência do sexo masculino com $54 \%(\mathrm{n}=409)$, a idade média foi de 56 anos. Foram prescritos 6870 medicamentos, sendo que 1727 (25\%) eram antimicrobianos, média de dois antibióticos prescritos por paciente. Obteve-se uma taxa de 52\% $(n=395)$ de interações medicamentosas entre os medicamentos antimicrobianos e de outras classes. O fármaco ciprofloxacino apresentou o maior número de interações, $42 \%(n=165)$, interagindo com 12 fármacos diferentes. Conclusão: as prescrições analisadas apresentaram um alto índice de potenciais interações medicamentosas. Dessa maneira, afirma-se que a presença do profissional farmacêutico inseridos nas equipes multiprofissionais e no processo de avaliação de prescrições no setor hospitalar, é de extrema importância e necessidade para diminuição dos riscos e aumento na segurança do paciente no uso de medicamentos.
\end{abstract}

Palavras-chave: Interação medicamentosa; Antimicrobianos; Farmacêutico clínico.

\section{Abstract}

Objective: to evaluate the potential drug interactions of antimicrobials in prescriptions of patients admitted to a hospital in the State of Pará, in 2019. Methodology: This is a qualitative-quantitative, retrospective and cross-sectional study, carried out by collecting data from medical records of patients who used antibiotics and were hospitalized in 2019 in a hospital in Pará. Drug interactions were assessed using the Micromedex database. Results: During the study period, $83 \%(n=762)$ of the patients who were admitted to the institution used antibiotics. There was a male 
prevalence of 54\% ( $\mathrm{n}=409)$, the average age was 56 years. 6870 drugs were prescribed, of which $1727(25 \%)$ were antimicrobials, an average of two antibiotics prescribed per patient. A 52\% rate $(\mathrm{n}=395)$ of drug interactions was obtained between antimicrobial drugs and other classes. The drug ciprofloxacin had the highest number of interactions, 42\% ( $\mathrm{n}=165)$, interacting with 12 different drugs. Conclusion: the prescriptions analyzed showed a high index of potential drug interactions. Thus, it is stated that the presence of the pharmaceutical professional inserted in the multiprofessional teams and in the process of evaluating prescriptions in the hospital sector, is of extreme importance and need to reduce risks and increase patient safety in the use of medications.

Keywords: Drug interaction; Antimicrobials; Clinical pharmacist.

\section{Resumen}

Objetivo: evaluar las posibles interacciones medicamentosas de los antimicrobianos en la prescripción de pacientes ingresados en un hospital del estado de Pará, en 2019. Metodología: Se trata de un estudio cualitativo-cuantitativo, retrospectivo y transversal, realizado mediante la recolección de datos de historias clínicas de pacientes que tomaron antibióticos y fueron hospitalizados en 2019 en un hospital de Pará. Las interacciones medicamentosas se evaluaron utilizando la base de datos Micromedex. Resultados: Durante el período de estudio, el 83\% ( $\mathrm{n}=762)$ de los pacientes que ingresaron a la institución utilizaron antibióticos. Hubo una prevalencia masculina del 54\% $(\mathrm{n}=409)$, la edad promedio fue de 56 años. Se prescribieron 6870 medicamentos, de los cuales 1727 (25\%) fueron antimicrobianos, un promedio de dos antibióticos prescritos por paciente. El fármaco ciprofloxacino tuvo el mayor número de interacciones, 42\% $(\mathrm{n}=165)$, interactuando con 12 fármacos diferentes. Conclusión: las prescripciones analizadas mostraron un alto índice de interacciones farmacológicas potenciales. Así, se afirma que la presencia del profesional farmacéutico insertado en los equipos multiprofesionales y en el proceso de evaluación de prescripciones en el sector hospitalario, es de suma importancia y necesidad para reducir riesgos y aumentar la seguridad del paciente en el uso de medicamentos.

Palabras clave: Interacción farmacológica; Antimicrobianos; Farmacéutico clínico.

\section{Introdução}

Interação medicamentosa (IM) é um processo que ocorre quando os efeitos e/ou toxicidade de um fármaco é modificado pela presença previa ou simultânea de outro fármaco (Santos et al., 2019; Alvim et al., 2015). As IM se classificam em interações físico-químicas, interações farmacocinéticas e interações farmacodinâmicas (Celdran et al.,2018).

No âmbito hospitalar, a grande maioria dos pacientes recebem múltiplos medicamentos, resultando no aumento do risco de interações medicamentosas de consequências graves para o paciente (Neves et al., 2015). As IM estão presentes na rotina hospitalar, e muitas não são identificadas por falta de avaliação das prescrições e ausência do farmacêutico clínico na equipe multiprofissional (Cortes et al., 2019). Dentre as interações importantes na prática clínica, destacam-se as ocorridas por meio do uso dos antimicrobianos, correspondendo a uma das classes de fármacos mais prescritas para uso terapêutico e profilático (Ministério da Saúde, 2018). Os Antimicrobianos são importantes na terapêutica, com o objetivo de curar infecções por microrganismos. No ambiente hospitalar, esses medicamentos são representados em $20 \%$ a $50 \%$ dos gastos totais com medicamentos (Piedade et al., 2015).

A análise de prescrições medicamentosas para investigação de possíveis interações farmacológicas é uma das atribuições do farmacêutico clínico, validada pela resolução de número 585 de 2013 do Conselho Federal de Farmácia (CFF, 2013). Dessa maneira, avaliar as condições de prescrições contendo antimicrobianos resultara no desenvolvimento de práticas que garantam o uso adequado e racional dos medicamentos, aumentando a efetividade e segurança do paciente (Rodrigues et al., 2017).

Sendo assim, o presente estudo tem como objetivo identificar e avaliar as potenciais interações medicamentosas em prescrições de pacientes internados que fizeram uso de antibióticos em um hospital no Estado do Pará, no ano de 2019.

\section{Metodologia}

Trata-se de estudo quali-quantitativo, retrospectivo e transversal, realizado em um hospital municipal no Estado do Pará, com análise de prescrições de pacientes internados no ano de 2019. 
O hospital é de pequeno porte, atendimento geral com porta de entrada de urgência e emergência e internação de clínica médica adulto. A instituição conta com 44 leitos ativos, dentre os quais 41 clínicos, 02 de cuidados semi-intensivos e 01 isolamento.

O levantamento de dados ocorreu através de prontuários de pacientes que foram hospitalizados no ano de 2019. Foram tabulados apenas os dados de pacientes internados acima de 18 anos e que fizeram uso de medicamentos antimicrobianos no período de estudo. Os dados extraídos dos prontuários foram: sexo; idade; diagnóstico através do código internacional da doença (CID); nomes dos medicamentos prescritos; quantidade de medicamentos prescritos. Para a análise e classificação das interações medicamentosas potenciais envolvendo os antimicrobianos, foi utilizada como fonte bibliográfica a base de dados "Drug Interactions Tool" do Micromedex® na versão 2.0. Conforme as informações disponíveis nas bases consultadas, as IM foram descritas quanto às reações adversas potenciais e classificadas quanto à gravidade (leve, moderada e grave), que leva em consideração o risco de efeitos clínicos, e quanto ao mecanismo de interação (farmacocinético, farmacodinâmico, físico-químico).

Como critério de inclusão considerou-se as prescrições médicas dos pacientes internados na instituição no período e com idade superior a 18 anos; prescrições com antimicrobianos; prontuários com prescrição médica interna. Os critérios de exclusão foram: prescrições de fitoterápicos e princípios ativos como vitaminas, sais minerais e componentes da dieta. Os dados foram registrados em planilhas no programa Microsoft Office Excel 2016. Os dados foram distribuídos em frequências absoluta e relativa e análise para avaliação de associações foi feita a partir dos testes de qui-quadrado e exato de Fisher, com nível de significância de $5 \%$ ( $\mathrm{p}<0,05)$.

A pesquisa foi aprovada junto ao Comitê de Ética em Pesquisa da Universidade Federal do Pará (UFPA) com número de CAAE: 37307620600000018.

\section{Resultados}

Foram coletadas prescrições de 919 pacientes. De acordo com os critérios utilizados, foram excluídas 157 prescrições. Dessa maneira, 83\% (n=762) das prescrições de pacientes internados no período de janeiro a dezembro de 2019 no hospital em estudo continham antimicrobianos.

Em relação ao sexo, verificou se que 54\% dos pacientes internados no período eram do sexo masculino. A faixa etária variou de 18 a 106 anos, com mediana igual a 36 e desvio padrão de 2,8. A prevalência foi da faixa etária de 18 até 59 anos, considerados adultos segundo a Organização Mundial de Saúde (OMS), com 54\% (n=409) e idosos acima de 60 anos de 46\% ( $\mathrm{n}=353)$. Através do CID, verificou-se que alguns dos diagnósticos mais prevalentes na causa de internações foram do sistema respiratório com $29 \%$ ( $\mathrm{n}=223)$, seguida de outras, tais como algumas doenças infecciosas e parasitárias com $18 \%$ ( $\mathrm{n}=140)$ e doenças do aparelho geniturinário com 17\% (n=127), esses resultados são apresentados na Tabela 1. 
Tabela 1. Perfil de pacientes internados em um hospital no Estado do Pará, no ano de 2019.

\begin{tabular}{|c|c|c|c|c|c|c|}
\hline \multirow{2}{*}{ Perfil } & \multicolumn{2}{|c|}{ Masculino } & \multicolumn{2}{|c|}{ Feminino } & \multicolumn{2}{|c|}{ Total } \\
\hline & $\%$ & $\mathbf{N}$ & $\%$ & $\mathbf{N}$ & $\%$ & $\mathbf{N}$ \\
\hline Gênero & $54 \%$ & 416 & $46 \%$ & 346 & $100 \%$ & 762 \\
\hline \multicolumn{7}{|l|}{ Faixa etária } \\
\hline $18-60$ anos & $55 \%$ & 226 & $45 \%$ & 183 & $54 \%$ & 409 \\
\hline Acima de 60 anos & $54 \%$ & 190 & $56 \%$ & 163 & $46 \%$ & 353 \\
\hline \multicolumn{7}{|l|}{ CID } \\
\hline A - Algumas doenças infecciosas e parasitárias & $59 \%$ & 82 & $41 \%$ & 58 & $18 \%$ & 140 \\
\hline B - Algumas doenças infecciosas e parasitárias & $69 \%$ & 11 & $31 \%$ & 5 & $2 \%$ & 16 \\
\hline $\mathrm{C}$ - Neoplasmas & $50 \%$ & 1 & $50 \%$ & 1 & $0,3 \%$ & 2 \\
\hline D - Doenças do sangue e dos órgãos hematopoiéticos e alguns transtornos imunitários. & $37 \%$ & 7 & $63 \%$ & 12 & $3 \%$ & 19 \\
\hline E - Doenças endócrinas, nutricionais e metabólicas. & $69 \%$ & 25 & $31 \%$ & 11 & $5 \%$ & 36 \\
\hline F - Transtornos mentais e comportamentais & $0 \%$ & 0 & $0 \%$ & 0 & $0 \%$ & 0 \\
\hline G -Doenças do sistema nervoso & $0 \%$ & 0 & $100 \%$ & 2 & $0,3 \%$ & 2 \\
\hline H -Doença dos olhos e anexos / Doenças dos ouvidos e da apófise mastoide & $50 \%$ & 1 & $50 \%$ & 1 & $0,3 \%$ & 2 \\
\hline F -Doença do aparelho circulatório & $45 \%$ & 10 & $55 \%$ & 12 & $3 \%$ & 22 \\
\hline J - Doença do aparelho respiratório & $54 \%$ & 121 & $46 \%$ & 102 & $29 \%$ & 223 \\
\hline $\mathrm{K}$ - Doença do aparelho digestivo & $34 \%$ & 18 & $56 \%$ & 35 & $7 \%$ & 53 \\
\hline L -Doença da pele e do tecido subcutâneo & $77 \%$ & 33 & $33 \%$ & 10 & $6 \%$ & 43 \\
\hline M - Doenças do sistema osteomuscular e do tecido conjuntivo. & $100 \%$ & 5 & $0 \%$ & 0 & $0,7 \%$ & 5 \\
\hline $\mathrm{N}$-Doenças do aparelho geniturinário. & $39 \%$ & 50 & $61 \%$ & 77 & $17 \%$ & 127 \\
\hline $\begin{array}{l}\text { R - Sintomas, sinais e achados anormais de exames clínicos e de laboratório, não } \\
\text { classificados em outra parte. }\end{array}$ & $40 \%$ & 6 & $60 \%$ & 9 & $1 \%$ & 15 \\
\hline S -Lesões, envenenamentos e algumas outras consequências de causas externas. & $100 \%$ & 4 & $0 \%$ & 0 & $0,5 \%$ & 4 \\
\hline T -Lesões, envenenamentos e algumas outras consequências de causas externas. & $78 \%$ & 40 & $22 \%$ & 11 & $7 \%$ & 51 \\
\hline \multirow[t]{2}{*}{ Não identificado } & $100 \%$ & 2 & $0 \%$ & 0 & $0,3 \%$ & 2 \\
\hline & & 416 & & 346 & & 762 \\
\hline
\end{tabular}

Fonte: Autores (2021).

Foram analisados 6870 medicamentos prescritos, dentre os 762 pacientes. Os antibióticos representaram $25 \%$ ( $\mathrm{n}=1727)$ das prescrições médicas, com média de dois antibióticos prescritos por paciente, sendo prescritos 18 tipos de antibióticos no período. A ceftriaxona apresentou maior número de prescrições, 37\% (n=631), sendo que 54\% (n=342) eram pacientes do sexo masculino e $46 \%(\mathrm{n}=289)$ feminino, representando o antimicrobiano mais prescrito para ambos os sexos (Tabela 2).

A clindamicina apresenta uma grande diferença em relação ao sexo, com maior número de prescrição 93\% (n=235) para homens, e apenas $7 \%(\mathrm{n}=18)$ para mulheres, podendo ser o tratamento de primeira escolha para infecção no trato respiratório superior (Tabela 2). 
Research, Society and Development, v. 10, n. 5, e29010515055, 2021

(CC BY 4.0) | ISSN 2525-3409 | DOI: http://dx.doi.org/10.33448/rsd-v10i5.15055

Tabela 2. Antimicrobianos prescritos no ano de 2019, em um hospital no Estado do Pará.

\begin{tabular}{lcccccc}
\hline \multirow{2}{*}{ Antibióticos Prescritos } & \multicolumn{2}{c}{ Masculino } & \multicolumn{2}{c}{ Feminino } & \multicolumn{3}{c}{ Total } \\
& $\%$ & $\mathbf{N}$ & $\%$ & $\mathbf{N}$ & $\mathbf{\%}$ & $\mathbf{N}$ \\
\hline Ceftriaxona & $54 \%$ & 342 & $46 \%$ & 289 & $37 \%$ & 631 \\
Clindamicina & $93 \%$ & 235 & $7 \%$ & 18 & $15 \%$ & 253 \\
Gentamicina & $46 \%$ & 97 & $54 \%$ & 115 & $12 \%$ & 212 \\
Metronidazol & $60 \%$ & 112 & $40 \%$ & 73 & $11 \%$ & 185 \\
Oxacilina & $66 \%$ & 114 & $34 \%$ & 59 & $10 \%$ & 173 \\
Ciprofloxacino & $51 \%$ & 41 & $49 \%$ & 40 & $5 \%$ & 81 \\
Ampicilina & $53 \%$ & 33 & $47 \%$ & 29 & $3 \%$ & 62 \\
Cefepime & $44 \%$ & 12 & $56 \%$ & 15 & $2 \%$ & 27 \\
Cloranfenicol & $46 \%$ & 12 & $54 \%$ & 14 & $1 \%$ & 26 \\
Azitromicina & $59 \%$ & 10 & $41 \%$ & 7 & $0,9 \%$ & 17 \\
Claritromicina & $47 \%$ & 8 & 53 & 9 & $0,9 \%$ & 17 \\
Vancomicina & $54 \%$ & 6 & $46 \%$ & 5 & $0,7 \%$ & 11 \\
Ceftadizima & $44 \%$ & 4 & $56 \%$ & 5 & $0,5 \%$ & 9 \\
Cefalotina & $86 \%$ & 6 & $14 \%$ & 1 & $0,4 \%$ & 7 \\
Imipeném & $57 \%$ & 4 & $43 \%$ & 3 & $0,4 \%$ & 7 \\
Amicacina & $40 \%$ & 2 & $60 \%$ & 3 & $0,3 \%$ & 5 \\
Penicilina Cristalina & $67 \%$ & 2 & $33 \%$ & 1 & $0,2 \%$ & 3 \\
Amoxicilina & $0 \%$ & 0 & $100 \%$ & 1 & $0,1 \%$ & 1 \\
\hline \multicolumn{1}{c}{ Total } & $\mathbf{6 0 \%}$ & $\mathbf{1 0 4 0}$ & $\mathbf{4 0 \%}$ & $\mathbf{6 8 7}$ & $\mathbf{1 0 0 \%}$ & $\mathbf{1 7 2 7}$ \\
\hline
\end{tabular}

Fonte: Autores (2021).

Foram identificadas 23\% ( $\mathrm{n}=395)$ de IM entre os antibióticos prescritos com outros medicamentos. As IM mais prevalentes entre as classes de antimicrobianos ocorreram entre: quinolonas e os hipoglicemiantes, opioides e glicorticóides; macrolídeos com opioides, glicocorticoides e anti-hipertensivos. O mecanismo de interação farmacodinâmico foi o mais frequente, presente em $57 \%(\mathrm{n}=223)$ das interações e farmacocinético em 43\% ( $\mathrm{n}=172)$ das interações. As interações classificadas como graves foram as mais prevalentes e corresponderam a $52 \%(n=206)$, sendo que destas, $60 \%(n=110)$ eram de prescrições de pacientes idosos.

O fármaco que apresentou maior número de IM foi o ciprofloxacino, com 42\% (n=165) e interagindo com 12 fármacos diferentes, seguido pela gentamicina com 17\% (n=67) de IM com cinco fármacos diferentes.

A interação medicamentosa entre o metronidazol e a cimetidina é classificada como moderada e foi representada por $28 \%(n=110)$ do total de interações medicamentosas, ocorrendo com maior prevalência em pacientes entre 18 a 59 anos.

Dentre os efeitos adversos mais predominantes que podem ser provocados pelas interações classificadas e identificadas como graves, estão o aumento do risco de prolongamento do intervalo do QT em 17\% (n=36), com frequência de 53\% (n=19) em prescrições de pacientes com idades entre 18 a 59 anos e ocorrendo prevalentemente nas prescrições com ciprofloxacino e metronidazol; Risco de depressão do SNC e depressão respiratória 27\% ( $\mathrm{n}=56$ ), com prevalência de 57\% $(\mathrm{n}=32)$ em pacientes adultos, com prescrição de ciprofloxacino e tramadol; aumento de risco de hipoglicemia ou hiperglicemia, $16 \%(n=33)$, com frequência de $61 \%(n=20)$ em pacientes idosos, ocorrendo com mais frequência entre o ciprofloxacino e insulina (Tabela 3). 


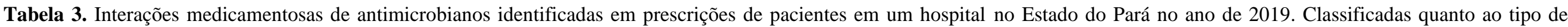
interação, mecanismo de interação e classificação em relação a faixa etária.

\begin{tabular}{|c|c|c|c|c|c|c|c|c|c|c|}
\hline \multirow{3}{*}{ Substância 1} & \multirow{3}{*}{ Substância 2} & \multirow{3}{*}{ Tipo de interação } & \multirow{3}{*}{ Mecanismo de interação } & \multirow{3}{*}{ Classificação } & \multirow{2}{*}{\multicolumn{4}{|c|}{ Interações }} & \multirow{2}{*}{\multicolumn{2}{|c|}{ Total }} \\
\hline & & & & & & & & & & \\
\hline & & & & & $\%$ & $\mathbf{N}$ & $\%$ & $\mathbf{N}$ & $\%$ & $\mathbf{N}$ \\
\hline Amicacina & Oxacilina & Farmacodinâmica & Inativação química do Aminoglicosídeo & Leve & $50 \%$ & 1 & $50 \%$ & 1 & $0,6 \%$ & 2 \\
\hline Amicacina & Furosemida & Farmacocinética & Toxicidade aditiva ou Sinérgica & Grave & $40 \%$ & 2 & $60 \%$ & 3 & $1 \%$ & 5 \\
\hline Ampicilina & Atenolol & Farmacocinética & Diminuição da Biodisponibilidade do atenolol & Leve & $0 \%$ & 0 & $100 \%$ & 1 & $0,2 \%$ & 1 \\
\hline Ampicilina & Gentamicina & Farmacodinâmica & Inativação química do Aminoglicosídeo & Leve & $17 \%$ & 1 & $83 \%$ & 5 & $1 \%$ & 6 \\
\hline Azitromicina & Claritromicina & Farmacodinâmica & Efeitos Aditivos no Intervalo QT & Grave & $0 \%$ & 0 & $100 \%$ & 2 & $0,6 \%$ & 2 \\
\hline Azitromicina & Aminofilina & Farmacocinética & Aumento das concentrações séricas de teofilina & Moderada & $25 \%$ & 1 & $75 \%$ & 3 & $1 \%$ & 4 \\
\hline Azitromicina & Ciprofloxacino & Farmacodinâmica & Risco aumentado de prolongamento do intervalo QT. & Grave & $56 \%$ & 5 & $44 \%$ & 4 & $2 \%$ & 9 \\
\hline Azitromicina & Morfina & Farmacodinâmica & Aumento da Morfina e depressão respiratória e SNC. & Grave & $33 \%$ & 1 & $67 \%$ & 2 & $1 \%$ & 3 \\
\hline Ciprofloxacino & Aminofilina & Farmacocinética & Diminuição da excreção da teofilina elevando níveis plasmáticos & Grave & $50 \%$ & 5 & $50 \%$ & 5 & $3 \%$ & 10 \\
\hline Ciprofloxacino & Dexametasona & Farmacodinâmica & Efeito aditivo do risco para ruptura de Tendão & Grave & $59 \%$ & 10 & $41 \%$ & 7 & $4 \%$ & 17 \\
\hline Ciprofloxacino & Fentanila & Farmacocinética & Inibição do metabolismo mediado por CYP3A4 & Grave & $0 \%$ & 0 & $100 \%$ & 3 & $1 \%$ & 3 \\
\hline Ciprofloxacino & Hidrocortisona & Farmacodinâmica & Risco aumentado de tendinite e ruptura do tendão & Grave & $67 \%$ & 10 & $33 \%$ & 5 & $4 \%$ & 15 \\
\hline Ciprofloxacino & H. de Aluminio & Farmacocinética & Diminuição da absorção de Ciprofloxacino devido á quelação & Moderada & $67 \%$ & 10 & $33 \%$ & 5 & $4 \%$ & 15 \\
\hline Ciprofloxacino & Insulina & farmacodinâmica & Risco de hipoglicemia grave, refratária e coma hiperglicêmico & Grave & $40 \%$ & 10 & $60 \%$ & 15 & $6 \%$ & 25 \\
\hline Ciprofloxacino & Metformina & Farmacodinâmica & Aumento de risco de hipoglicemia ou hiperglicemia, Hipertensão & Grave & $38 \%$ & 3 & $62 \%$ & 5 & $2 \%$ & 8 \\
\hline Ciprofloxacino & Metronidazol & Farmacocinética & Prolongamento Aditivos do intervalo QT & Grave & $58 \%$ & 8 & $42 \%$ & 6 & $4 \%$ & 14 \\
\hline Ciprofloxacino & Midazolam & Farmacocinética & Inibição do metabolismo mediado por CYP3A4 & Grave & $0 \%$ & 0 & $100 \%$ & 2 & $0,6 \%$ & 2 \\
\hline Ciprofloxacino & Prometazina & Farmacodinâmica & Risco aumentado do Intervalo QT & Grave & $100 \%$ & 4 & $0 \%$ & 0 & $1 \%$ & 4 \\
\hline Ciprofloxacino & Tramadol & Farmacodinâmica & Risco de depressão do SNC e depressão respiratória & Grave & $58 \%$ & 30 & $42 \%$ & 22 & $13 \%$ & 52 \\
\hline Ceftazidima & Cloranfenicol & Farmacodinâmica & Antagonismo diminuição da eficácia da Ceftazidima & Leve & $50 \%$ & 2 & $50 \%$ & 2 & $1 \%$ & 4 \\
\hline Claritromicina & Aminofilina & Farmacodinâmica & Toxicidade de Teofilina, náuseas, vômitos, palpitações & Leve & $25 \%$ & 1 & $75 \%$ & 3 & $1 \%$ & 4 \\
\hline Claritromicina & Dexametasona & Farmacocinética & Indução do Metabolismo do substrato mediado pelo CYP3A4 & Grave & $100 \%$ & 1 & $0 \%$ & 0 & $0,2 \%$ & 1 \\
\hline Claritromicina & Losartana & Farmacocinética & Inibição do Metabolismo mediado pelo CYP3A4 & Grave & $100 \%$ & 1 & $0 \%$ & 0 & $0,2 \%$ & 1 \\
\hline Claritromicina & Morfina & Farmacodinâmica & Aumenta o risco de depressão respiratória e do SNC & Grave & $100 \%$ & 1 & $0 \%$ & 0 & $0,2 \%$ & 1 \\
\hline Claritromicina & Nifedipino & Farmacodinâmica & Risco de Hipotensão, bradicardia ou lesão renal aguda & Grave & $50 \%$ & 1 & $50 \%$ & 1 & $0,6 \%$ & 2 \\
\hline Claritromicina & Prometazina & Farmacodinâmica & Aumento do risco de prolongamento do intervalo do QT. & Grave & $0 \%$ & 0 & $100 \%$ & 2 & $0,6 \%$ & 2 \\
\hline Claritromicina & Tramadol & Farmacocinética & Inibição do metabolismo de Tramadol mediado pelo CYP3A4 & Grave & $67 \%$ & 2 & $33 \%$ & 1 & $0,8 \%$ & 3 \\
\hline Cloranfenicol & Ferro 111 & Farmacocinética & Pode resultar na diminuição da eficácia do Ferro & Leve & $100 \%$ & 3 & $0 \%$ & 0 & $0,8 \%$ & 3 \\
\hline Gentamicina & Furosemida & Farmacodinâmica & Aumento de nefro toxicidade e Ototoxicidade & Grave & $32 \%$ & 10 & $68 \%$ & 21 & $8 \%$ & 31 \\
\hline Gentamicina & Digoxina & Farmacocinética & Concentrações de aumento de Digoxina & Leve & $0 \%$ & 0 & $100 \%$ & 2 & $0,6 \%$ & 2 \\
\hline Gentamicina & Oxacilina & Farmacodinâmica & Perda de eficácia aminoglicosida & Leve & $60 \%$ & 15 & $40 \%$ & 10 & $6 \%$ & 25 \\
\hline Gentamicina & Penicilina & Farmacodinâmica & Perda de eficácia aminoglicosida & Leve & $33 \%$ & 1 & $67 \%$ & 2 & $0,8 \%$ & 3 \\
\hline Imipenem & Aminofilina & Farmacodinâmica & Pode resultar em toxicidade por teofilina & Grave & $0 \%$ & 0 & $100 \%$ & 1 & $0,2 \%$ & 1 \\
\hline Metronidazol & Prometazina & Farmacodinâmica & Risco de prolongamento e arritmias do intervalo QT. & Grave & $40 \%$ & 2 & $60 \%$ & 3 & $1 \%$ & 5 \\
\hline Metronidazol & Cimetidina & Farmacocinética & Inibição do metabolismo pelo CYP450 de MTZ por Cimetidina & Moderada & $68 \%$ & 70 & $32 \%$ & 40 & $28 \%$ & 110 \\
\hline & & & & Total & & 211 & & 184 & & 395 \\
\hline
\end{tabular}

Fonte: Autores (2021) 
Constatou-se que $86 \%(\mathrm{n}=656)$ das prescrições haviam cinco ou mais medicamentos prescritos, classificado como polifarmácia. O menor número de medicamentos em prescrição foi dois e o maior 20, com média de nove medicamentos prescritos por paciente. As 395 interações encontradas foram todas referente ao grupo de prescrições com polifarmácia, representando $60 \%$ destas. Tal valor é significativamente maior que o outro grupo de prescrições ( $p<0,0001$ ), o que indica que quanto maior o número de medicamentos prescritos, maior a probabilidade de ocorrer interação entre os medicamentos.

\section{Discussão}

No presente estudo obteve-se predominância de pacientes internados do sexo masculino no ano de 2019 no hospital em estudo, corroborando com a pesquisa de Murtaza et al. (2016), no qual 55\% dos pacientes em sua pesquisa eram do sexo masculino e 45\% feminino, com idade média de 62 anos. O de Mousavi \& Ghanbari (2017) também evidencia a prevalência de internações masculinas com 59\% e média de idade de 61 anos. Outros estudos realizados no Brasil, também destacam a prevalência de homens internados com frequências acima de 55\%, tal fator tem sido justificado devido barreiras sociais e culturais dos homens que procuram o serviço de saúde apenas quando o quadro se torna crítico (Cedraz \& Santos, 2014; Nascimento et al., 2018; Reis et al., 2016). Os estudos citados apresentam diferenças em relação à faixa etária e os diagnósticos encontrados, com prevalência de idosos, enquanto esta pesquisa evidencia uma população adulta internada. Os diagnósticos mais frequentes apresentados em estudos com perfil de pacientes adultos e idosos são doenças do sistema cardiorrespiratório e circulatório. Esses dados podem variar e gerar resultados diferentes desta pesquisa, devido localidade, perfil sócio econômico da população, clima e organização das redes de atenção à saúde conforme observados em outros estudos (Carvalho, 2010; Cedraz \& Santos, 2014; Guia et al., 2015; Murtaza et al., 2016; Nascimento et al., 2018; Perão et al., 2017; Reis et al., 2016; Rodriguez et al., 2016; Araújo et al., 2019; Ueno et al., 2018; Leal, 2020).

$\mathrm{Na}$ instituição em estudo, a escolha do medicamento antimicrobiano foi baseada na clínica do paciente e em exames laboratoriais com achados de sinais de infecção, mas não de identificação do microrganismo, ou seja, o início do tratamento com antibióticos foi empírico. Aconselha-se que a escolha do medicamento antibiótico seja baseada por testes de efetividade microbiológica para microrganismos recolhidos do paciente, para evitar risco de resistência bacteriana (Vieira \& Vieira, 2017). A ceftriaxona foi identificada como o antibiótico mais prescrito na unidade hospitalar em estudo corroborando com um estudo realizado em um hospital na Etiópia, com prevalência de 58\% de prescrições de Ceftriaxona (Muhammed \& Nasir, 2020). Em outro estudo conduzido em três hospitais localizados no município de Mosh, no nordeste da Tanzânia a ceftriaxona e o metronidazol foram os antibióticos mais comumente prescritos. (Horumpende et al., 2020). A prevalência do uso da ceftriaxona pode ser justificada por ser um medicamento de amplo espectro que pode ser utilizada na terapia de uma gama de infecções, sendo utilizada como primeira escolha para casos como: meningite bacteriana aguda, pneumonia adquirida pela comunidade, infecções intra-abdominais complicadas, infecções intra-abdominais complicadas, pneumonia hospitalar adquirida; pielonefrite, entre outros (Sonda et al., 2019).

A frequência de interações medicamentosas graves neste estudo correspondeu a 52\%, semelhante a dois estudos realizados no continente asiático (Noor et al., 2019; Rabba et al., 2020). No Brasil, um estudo de Alvim et al. (2015) em um hospital no estado de Minas Gerais, constatou uma frequência acima de 50\% em IM consideradas graves. Considerando os resultados negativos e com risco de morte de interações medicamentosas, a presença do farmacêutico clínico nos hospitais se torna uma alternativa eficaz na redução dos riscos e prevenção de eventos adversos graves, garantindo o tratamento seguro e eficaz ao paciente internado (Freitas et al., 2019).

O metronidazol e ciprofloxacino apresentaram o maior número de interações com outros medicamentos e são destacados como fármacos de interação mais frequente. No estudo de Sanchez-Lopez (2016) no México, foram prevalentes as 
interações encontradas em relação ao uso desses dois fármacos e a classe das fluoroquinolonas no geral. Esses medicamentos causam prolongamento do intervalo QT e seu uso simultâneo aumenta o risco deste efeito colateral, e por isso deve-se dar atenção a essa interação (Khan et al., 2017). As fluoroquinolonas são antibióticos muito utilizados na prática clínica devido ao seu amplo espectro, estando entre os antibacterianos que representam as classes de anti-infecciosos que estão mais ligados ao aumento do intervalo QT (Lemos et al., 2021; Gnanapandithan et al., 2021).

O número de medicamentos prescritos por paciente apresentou uma elevada frequência com média de nove medicamentos por paciente, considerado polimedicação corroborando com uma pesquisa realizada na Itália o qual os pacientes estavam em terapia com média de oito medicamentos (Piccoliori et al., 2021). Estudo retrospectivo com prescrições hospitalares apresentaram resultados semelhantes aos deste trabalho, as potenciais interações medicamentosas eram descritas em prescrições com cinco ou mais medicamentos, sugerindo associação entre a polimedicação e ocorrência de interação medicamentosa (Martocchia et al., 2020; Arai et al., 2019). A polimedicação é um dos principais problemas na segurança do paciente, pois aumenta as chances de evento adverso devido erros de medicação e interações medicamentosas (Donaldson $e t$ al., 2017).

\section{Conclusão}

A identificação e análise das interações medicamentosas com antimicrobianos nesse estudo demostraram um elevado número de interações, sendo as graves mais prevalentes. Os resultados colhidos nessa investigação evidenciaram a necessidade de informação para a equipe a respeito dos medicamentos utilizados na instituição. A partir dessas colocações, ressalta-se a necessidade da presença do farmacêutico na equipe multiprofissional hospitalar, contribuindo para eficácia da terapêutica medicamentosa e segurança do paciente.

\section{Referências}

Alvim, M. M., Silva, L. A., Leite, I. C.G. \& Silvério, M. S. (2015). Eventos adversos por interações medicamentosas potenciais em unidade de terapia intensiva de um hospital de ensino. Revista Brasileira de Terapia Intensiva, 27 (4), 353-359.

Arai, S., Ishikawa, T., Kato, H., Koshizaka, M., Maezawa, Y., Nakamura, T., Suzuki, T., Yokote, K. \& Ishii I. (2019). Multidrug use positively correlates with high-risk prescriptions in the Japanese elderly: a longitudinal study. Journal of Pharmaceutical Health Care and Sciences, 5:20.

Araújo, L. U., Santos, D. F., Bodevan, E. C., Cruz, H. L., Souza, J. \& Silva-barcellos, N. M. (2019) Patient safety in primary health care and polypharmacy: cross-sectional surve,y among patients with chronic diseases. Revista Latino-Americana de Enfermagem, $27,3217$.

Brasil. Conselho Federal de Farmácia. Resolução n585 de 29 de agosto de 2013. Regulamenta as atribuições clínicas do farmacêutico e dá outras providências. Diário Oficial da União, Brasília; $2013^{\text {a }}$

Brasil. Ministério da Saúde. Saúde e Política externa: os 20 anos da Assessoria de Assuntos Internacionais de Saúde (1998-2018). $1^{\text {a }}$ nd ed. Brasília: Ministério da Saúde, 2018; 364 p.

Carvalho, A. M. R. (2010). Análise da prescrição de pacientes utilizando sonda enteral em um hospital universitário do Ceára. Rev. Bras. Farm. Hosp. Serv. Saúde, 1 (1), 17-22.

Cedraz K. N. \& Santos, M. C. (2014). Identificação e caracterização de interações medicamentosas em prescrições médicas da unidade de terapia intensiva de um hospital público da cidade de Feira de Santana, BA. Revista Sociedade Brasileira de Clínica Médica, 12 (2), 1-7.

Celdran, L. M. M., Ferrando, J. G. \& Royo, L. M. (2018). ¿Conocemos todas las interacciones farmacológicas?: el transportador OATP1B1. Farmacéuticos comunitarios, $10(4), 29-32$.

Cortes, A. L. B., Silvino, Z. R., Santos, F. B. M., Pereira, J. A. C. \& Tavares, G. S.(2019). Prevalência de interações medicamentosas envolvendo medicamentos de alta-vigilância: estudo transversal. Revista Mineira de Enfermagem, 23, 1226.

Donaldson, L.J., Kelley, E. T., Dhingra-Kumar, N. Kieny, M. P. \& Sheikh, A. (2017). Medication without harm: WHO’s Third Global Patient Safety Challenge. The Lancet, 389 (10080), 1680-1681.

Freitas, D., Silva, J. \& Scalco, T (2019). Resultados negativos associados à medicação em idosos hipertensos e diabéticos. Journal Health NPEPS, 4 (2), 118131. 
Gnanapandithan, K., Karthik, N. \& Gerber, J. (2021). Methadone, Metoclopramide and Metronidazole Interaction Causing Torsades de Pointes. Clinics and practice, $11(1), 101-105$

Guia, C.M., Biondi, R. S., Sotero, S., Lima, A. A., Almeida, K. J. Q. \&Amorim, F. F. (2015). Perfil epidemiológico e preditores de mortalidade de uma unidade de terapia intensiva geral de hospital público do distrito federal. Revista Comunicação em Ciências da Saúde, 26 (1/2), 9-19.

Horumpende, P. G., Mshana, S. E., Mouw, E. F., Mmbaga, B. T., Chilongola, J. O. \& Mast, Q. (2020). Point prevalence survey of antimicrobial use in three hospitals in North-Eastern Tanzania. Antimicrobial resistance and infection control, 9 (1), 149.

Khan, Q., Ismail, M. \& Khan, S. (2017). Frequency, characteristics and risk factors of QT interval prolonging drugs and drug-drug interactions in cancer patients: a multicenter study. BMC pharmacology \& toxicology, 18 (1), 75.

Leal, L. F., Cousin, E., Bidinotto, A. Bacelo., Sganzerla, D., Borges, R. B., Malta, D. C., Ikuta, K. \& Pizzol, T. S. D. (2020). Epidemiology and burden of chronic respiratory diseases in Brazil from 1990 to 2017: analysis for the Global Burden of Disease 2017 Study. Revista Brasileira de Epidemiologia, 23 (1), 200031 .

Lemos, L., Goulão, M.C., Figueiredo, D., Santos, J., Morgano, S. \& Morgano, M. (2021).Revision: Medicamentos com potencial para prolongarem o intervalo QT e precauções a ter na prática clínica com a sua utilização.Ilafhar, 25 (1), 41-47.

Martocchia, A., Spuntarelli, V., Aiello, F., Meccariello, A. L., Proietta, M., Del Porto, F., Di Rosa, R., Salemi, S., Rocchietti March, M., Laganà, B., Martelletti, P. \& Sesti, G. (2020). Using INTERCheck® to Evaluate the Incidence of Adverse Events and Drug-Drug Interactions in Out- and Inpatients Exposed to Polypharmacy. Drugs Real World Outcomes, 7 (3), 243-249.

Mousavi, S. \& Ghanbari, G. (2017). Potential drug-drug interactions among hospitalized patients in a developing country. Caspian Journal of Internal Medicine, 8 (4), 282-288.

Muhammed, O. S. \& Nasir, B. B. (2020). Drug Use Evaluation of Ceftriaxone in Ras-Desta Memorial General Hospital, Ethiopia. Drug. Healthcare and Patient Safety, 12, 161-168.

Murtaza, G., Khan, M. Y., Azhar, S., Khan, S. A. \& Khan, T. M. (2016). Assessment of potential drug-drug interactions and its associated factors in the hospitalized cardiac patients. Saudi Pharmaceutical Journal, 24 (2), 220-225.

Nascimento, M. S. M., Nunes, E. M., Medeiros, R. C., Souza, W. I. M., Filho, L. F. S. \& Alves, E. S. R. C. (2018). Perfil epidemiológico de pacientes em unidade de terapia intensiva adulto de um hospital regional paraibano. Temas em Saúde, 18 (1), 247- 265.

Neves, C. \& Colet, C. (2015). Perfil de uso de antimicrobianos e suas interações medicamentosas em uma uti adulto do rio grande do sul. Revista de Epidemiologia e Controle de Infecção, 5 (2), 65-71.

Noor, S., Ismail, M. \& Khan, F. (2019). Potential Drug-Drug Interactions in Patients With Urinary Tract Infections: A Contributing Factor in Patient and Medication Safety. Frontiers in pharmacology, 10, 1032

Perão, O., Bub, M., Zandonadi, G. \& Martins, M. (2017). Características sociodemográficas e epidemiológicas de pacientes internados em uma unidade de terapia intensiva de adultos [Sociodemographic and epidemiological characteristic of patients in an adult intensive care unit. Revista Enfermagem UERJ, 25, 7736.

Piccoliori, G., Mahlknecht, A., Sandri, M., Valentini, M., Vögele, A., Schmid, S., Deflorian, F., Engl, A., Sönnichsen, A. \& Wiedermann, C. (2021). Epidemiology and associated factors of polypharmacy in older patients in primary care: a northern Italian cross-sectional study. BMC geriatrics, 21 (1), 197.

Piedade, D. V., Silva, L. A. F., Lemos, G. S., Valasque, JR. G. L. \& Lemos, L.B. (2015). Interações medicamentosas potencias em prescrições, contendo antimicrobianos de uso restrito, de pacientes internados em um hospital no interior da Bahia. Medicina (Ribeirão Preto), 48 (3), $295-307$.

Rabba, A. K., Abu Hussein, A. M., Abu Sbeih, B. K. \& Nasser, S. I. (2020). Assessing Drug-Drug Interaction Potential among Patients Admitted to Surgery Departments in Three Palestinian Hospitals. BioMed Research International, 2020, 9634934.

Rêgo, M. M. \& Comarella, L. (2015). O papel da análise farmacêutica da prescrição médica hospitalar. Caderno Saúde e Desenvolvimento, 7 (4), 17-31.

Reis, L. C. C., Gabarra, L. M. \& Moré, C. L. O. O. (2016). As repercussões do processo de internação em UTI adulto na perspectiva de familiares. Temas em Psicologia, 24 (3), 815-828.

Rodrigues, A. P., Dambrós, B. P. Santin, N. C. \& Frighetto, M. (2017). Análise das prescrições de antibióticos dispensados para crianças numa farmácia de um município Catarinense. Revista Interdisciplinar, 10 (4), 69-76.

Rodriguez, A.H., Bub, M.B., Perão, O.F., Zandonadi, G. \& Rodriguez M. de J.(2016) Epidemiological characteristics and causes of deaths in hospitalized patients under intensive care. Revista Brasileira de Enfermagem, 69 (2), 229-234.

Sánchez-López, V. A., Brennan-Bourdon, L. M., Rincón-Sánchez, A. R., Islas-Carbajal, M. C., Navarro-Ruíz, A. \& Huerta-Olvera, S. G.(2016). Prevalence of potential drug-drug interactions in hospitalized surgical patients. The Journal of Pharmacy and Pharmacology, 4 (12), 658-666.

Santos, J. S., Giordani, F. \& Rosa, M. L. G. (2019). Interações medicamentosas potenciais em adultos e idosos na atenção primária. Ciência \& saúde coletiva, $24(11), 4335-4344$

Sonda, T. B., Horumpende, P. G., Kumburu, H. H., van Zwetselaar, M., Mshana, S. E., Alifrangis, M., Lund, O., Aarestrup, F. M., Chilongola, J. O., Mmbaga, B. T. \& Kibiki, G. S. (2019). Ceftriaxone use in a tertiary care hospital in Kilimanjaro, Tanzania: A need for a hospital antibiotic stewardship programme. PloS One, 14 (8), 0220261. 
Research, Society and Development, v. 10, n. 5, e29010515055, 2021

(CC BY 4.0) | ISSN 2525-3409 | DOI: http://dx.doi.org/10.33448/rsd-v10i5.15055

Ueno, E., Koffke, M. \& Voigt, V. R. (2018). Rofile of hospitalized patients under enteral therapy. Braspen Journal, 33 (2), 194-198.

Vieira, P. N. \& Vieira, S. L. V. (2017). Uso irracional e resistência a antimicrobianos em hospitais. Arquivos de Ciências da Saúde da UNIPAR, 21 (3), 209213. 